\title{
Comparison of a Pain Pump versus Injectable Medication for Analgesia in Knee Arthroscopy
}

\section{——A Randomized Double Blind Controlled Study}

\author{
Albert W. Pearsall", Sudhakar G. Madanagopal, Kshitij Agrawal \\ Department of Orthopaedic Surgery, University of South Alabama, Mobile, USA. \\ Email: *apearsal@usouthal.edu
}

Received July $3^{\text {rd }}, 2012$; revised July $24^{\text {th }}, 2012$; accepted August $10^{\text {th }}, 2012$

\begin{abstract}
As arthroscopic knee procedures have evolved, so has the management of post-operative pain. Traditional pain management included intravenous narcotics, which often resulted in nausea and increased sedation. In an effort to reduce post-operative pain and minimize complications, the concept of multimodal analgesia evolved, including subcutaneous infiltration of local anesthetic, ketorolac and/or narcotic. We studied the effect upon post-operative pain of combining ropivacaine, ketorolac and morphine injected as a one-time dose compared to the same mixture plus an intraarticular pump administering ropivacaine for 24 hours. Our findings showed that a single subcutaneous and intraarticular injection of ketorolac, morphine and ropivacaine was as effective as this solution plus a 24-hour intraarticular ropivacaine pain pump for post-operative pain relief after outpatient arthroscopic knee surgery. No clinical or radiographic signs of chondrolysis were seen in the knee with the use of intraarticular ropivacaine at an average of 18 months post-operatively.
\end{abstract}

Keywords: Pain Pump; Anesthetics; Knee Arthroscopy

\section{Introduction}

Outpatient surgery is one of the fastest growing fields in health care. One contributing factor to its success is pain control in the immediate post-operative period. In an effort to reduce post-operative pain and minimize potential complications, authors have reported the use of multimodal analgesia, including wound infiltration of local anesthetic, ketorolac and/or narcotics [1-4]. The postoperative use of a subcutaneous or intraarticular infiltration device (pain pump) for 24 hours or greater has also been reported with improved outcomes [5,6].

The purpose of the current study was to evaluate the added benefit of a 24-hour intraarticular pain pump containing ropivacaine when used in conjunction with local wound and joint infiltration with medication. In addition, the authors wished to evaluate the safety of continuously administered ropivacaine within the knee.

\section{Materials and Methods}

\subsection{Study Participants}

All patients who underwent knee partial meniscectomy and/or chondroplasty were invited to participate in the

${ }^{*}$ Corresponding author. study. Exclusion criteria were the following: 1) surgical procedure within the same joint within 90 days; 2) any acute/chronic knee infection; 3) diagnosis of complex regional pain syndrome; 4) known allergy to any of the study drugs; 5) documented history of narcotic use; 6) score of less than 2 standard deviations on the SF-12 mental component; 7) major systemic or cardiac illness and 8) less than 18 years of age. The study protocol was approved by our University Institutional Review Board and registered at www.clinicaltrials.gov. The protocol number is NCT01242644. All subjects were given written informed consent to participate in the study.

\subsection{Study Design}

After a study briefing, each participant signed a written consent and underwent clinical assessment and completed a demographic questionnaire, the Western Ontario and McMaster Universities Arthritis Index (WOMAC) and a SF-12 Health Survey. At surgery, each subject was randomly assigned to one of three treatment groups (Figure 1). All groups received $30-\mathrm{mL}$ of Naropin (ropivacaine) $(0.5 \%, 5 \mathrm{mg} / \mathrm{mL}), 30 \mathrm{mg}$ of ketorolac $(30 \mathrm{mg} / 1$ $\mathrm{mL})$ and $8 \mathrm{mg}$ of morphine sulfate $(8 \mathrm{mg} / 2-\mathrm{mL})$ totaling $33-\mathrm{mL}$ of injectable solution. The solution was injected 


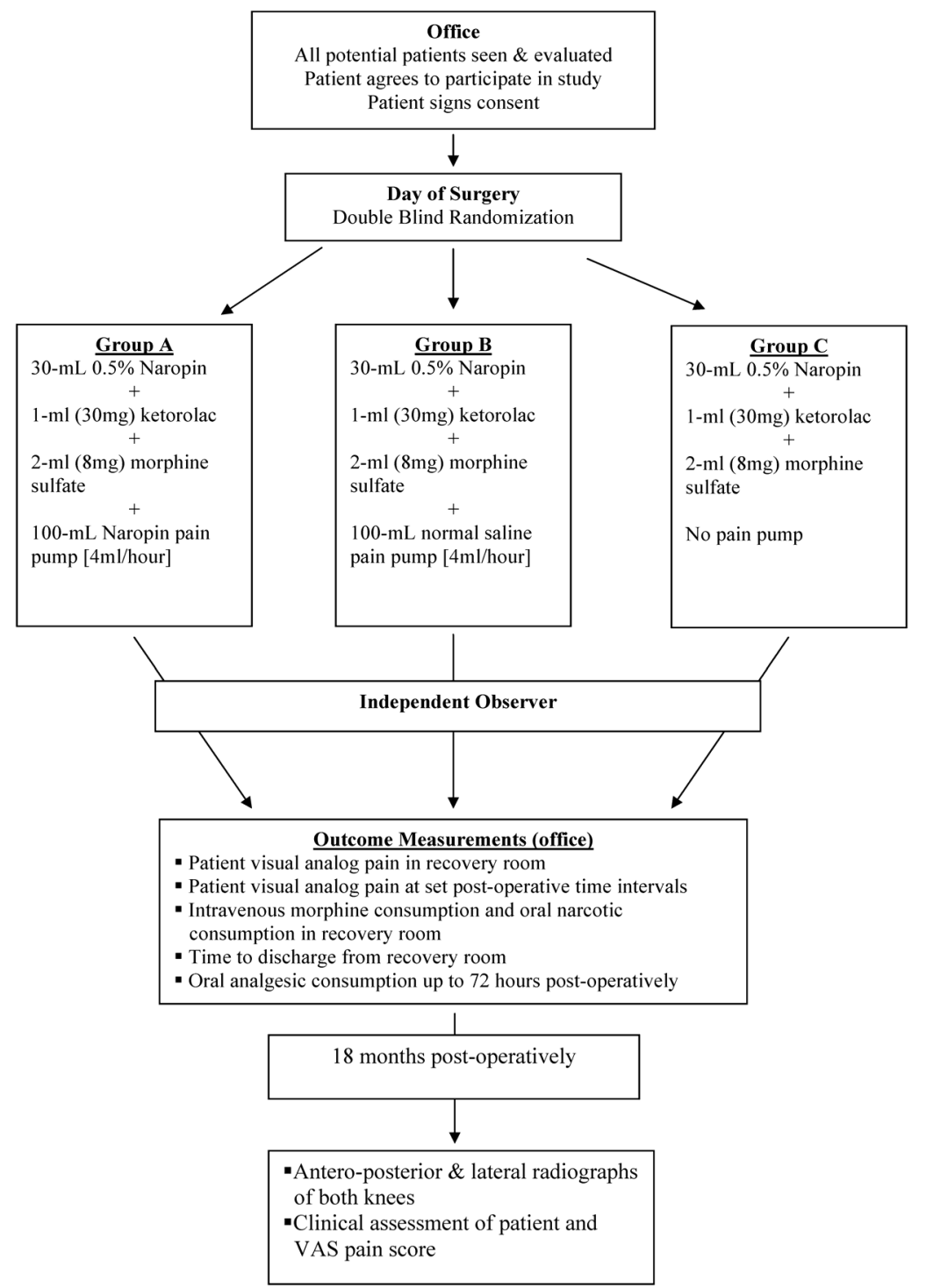

Figure 1. Study outline of the current investigation. All patients were seen in the office, consented for study enrollment and randomized on the day of surgery. Outcome measures were recorded by an independent observer for 48 to 72 hours after surgery. Standardized digital radiographs were obtained of all study patients at 9 months post-operatively.

subcutaneously and intramuscularly at the portalsites. Ten milliliters was reserved for injection into the intraarticular space. Group A received an intraarticular pain pump containing $100-\mathrm{mL}$ of Naropin (ropivacaine) $(0.5 \%, 5$ $\mathrm{mg} / \mathrm{mL}$ ) set at 4-mL/hour. Group B received an identical intraarticular pain pump containing normal saline set at 4-mL/hour. Group C did not receive a pump. In the initial phase of the study, all pain pumps were manufactured by Zimmer (Zimmer Inc., Warsaw, Indiana). Midway through the study, these devices were no longer available. Upon approval by our University Institutional Review Board, a device utilizing the same reservoir and flow rate was used from a different manufacturer (Stryker Orthopaedics, Mahwah, New Jersey).

A randomization table was created with SPSS 10.04 software (SPSS, Chicago, Illinois). All study participants, research assistants, clinic nurses and physiotherapists were blinded to the subject's group. Upon enrollment in the study, the subject was randomized to a group. The study coordinator alerted the operating room staff 24 hours prior to the procedure that a patient was a candidate for the study protocol. At surgery, if the surgeon determined that the procedure met the inclusion criteria, an envelope was opened in the operating room and the patient assigned to one of the three groups. The operating room staff prepared the $33-\mathrm{mL}$ syringe for all Groups and the $100-\mathrm{mL}$ pain pump for Groups A and B. All patients in Groups A and B removed their intraarticular pumps 24 hours after surgery. The surgeon and operating room staff were not blinded to the subjects' group assignment. 
All research and clinical personnel involved in the subjects' post-operative care were blinded.

\subsection{Surgery}

All patients received general anesthesia during surgery. Prior to the skin incision, $10 \mathrm{cc}$ of $1 \%$ lidocaine with 1:100,000 epinephrine was injected into all arthroscopy portals and the intraarticular space. Standard knee arthroscopy was performed. Upon completion, the surgeon was handed a labeled $33-\mathrm{mL}$ syringe containing the medication and a pain pump (Groups A and B). The surgeon injected the medication subcutaneously and intramuscularly at the portal sites with ten milliliters injected into the intraarticular space. For Groups A and B, the pump catheter tip was placed intraarticularly through the lateral suprapatellar space. The placement of the catheter tip within the knee joint was confirmed arthroscopically.

\subsection{Outcome Measures}

Preoperative pain level and range of motion was recorded in the recovery room and all patients were observed in the recovery room until discharge. The postoperative pain level was recorded during the first hour after surgery and at 8 hours, 24 hours, 48 hours and 72 hours post-operatively. All patients estimated their postoperative pain level on a 10-point visual analog scale (VAS). Prior to discharge, the study coordinator instructed the patient to remove the pain pump after 24 hours (Groups A and B) and complete a study form documenting their pain level, the number of pain pills taken and any complications that occurred during the next 72 hours. All discharged patients were given a prescription for 10 $\mathrm{mg}$ ketorolac and hydrocodone $7.5 \mathrm{mg} /$ acetaminophen $500 \mathrm{mg}$. Within 48 hours post-operatively, all study patients began physical therapy. Knee range of motion was documented by a physiotherapist at 48 and 72 hours after surgery.

At a minimum of 12 months post-operatively, all patients underwent radiographic examination of their operated knee including standing antero-posterior (AP) and skyline views taken at $45^{\circ}$ of knee flexion. Using a computer measurement system (Philips Healthcare, Andover, MA), the shortest distance between the femoral condyle and the tibial plateau on the AP radiograph and between the medial and lateral facets of the patella and the adjacent trochlea on the skyline view were recorded in millimeters. The sum of the four distances was recorded as the composite score, which was similarly calculated for each study patient pre-operatively. All films were read twice on two different occasions by an orthopaedic surgeon blinded to the patient's group and the average composite score used for analysis. Each patient's preoperative and 12 months post-operative scores were analyzed using a paired t-test.

The primary outcomes measured were: 1) total milligrams of intravenous morphine and/or number of narcotic tablets required by the patient in the recovery room prior to discharge or admission to the hospital; 2) time from surgery to discharge measured in minutes from admission to recovery room; 3) number of narcotic pills used within the first 72 hours; 4) the patient's pain level as measured on a visual analog scale.

\subsection{Statistical Analyses}

With an alpha error of 0.05 and power of $80 \%$, it was determined that 14 patients would be required in each group to detect a significant difference between groups of 3 narcotic pills or 2 points on a visual analog pain scale. Once the data was collected, it was entered into a database and analyzed using SPSS software. Analysis of variance (ANOVA) was performed between the 3 groups for all continuous outcome variables. Kruskal Wallis analysis was used for nonparametric variables. Chi square tests were used for all categorical values. Post hoc Tukey tests were performed to determine differences between individual groups. Correlation analysis was performed to analyze any relationship between independent and dependent variables. Significance for all analysis was set at $\mathrm{p} \leq 0.05$.

\section{Results}

Forty-eight patients were enrolled in the study [pain pump (16), saline pump (17), no pump (15)]. The numbers in each group were not statistically different $(p=$ 0.1 ). There were 24 males and 24 females, with a mean age of 51.2 years. There was no statistical difference between the mean age of male and female patients $(p=$ 0.9 ). The distribution of male to female was also not significant in any group $(p=0.9)$. The average body mass index for the study group was 32.6. No difference in BMI was noted among the three study groups $(p=0.33)$. Fifty-two percent of patients underwent chondroplasty of the medial or lateral femoral condyle, while $72 \%$ of patients underwent partial meniscectomy of the medial and/or lateral meniscus. There was no statistical difference noted in activity level in any group. There was no significant difference among groups regarding who had undergone a previous surgery on the study $\operatorname{limb}(\mathrm{p}=0.47)$ (Table 1). The pre-operative WOMAC score $(\mathrm{p}=0.8)$, SF-12 physical score $(\mathrm{p}=0.7)$, SF 12 mental score $(\mathrm{p}=$ $0.9)$ and preoperative knee range of motion $(p=0.8)$ was not found to be statistical significant among the groups. The time spent in recovery room prior to discharge was not significantly different among groups $(p=0.46)$.

The average post-operative pain scores for the recorded time intervals are shown (Table 2). On the day of 
Table 1. Study group demographics.

\begin{tabular}{|c|c|c|c|c|}
\hline Demographics & Group A [pain pump] & Group B [saline pump] & Group C [no pump] & $\mathrm{p}$ value \\
\hline Age [years] & 51 & 51 & 49.9 & 0.9 \\
\hline Sex [male/female] & 10 male $/ 6$ female & 7 male/10 female & 7 male $/ 8$ female & 0.35 \\
\hline Previous surgery & $3(20 \%)$ & $2(12 \%)$ & $1(6 \%)$ & 0.47 \\
\hline
\end{tabular}

Table 2. VAS pain scores for the three groups at different time intervals.

\begin{tabular}{cccc}
\hline Time interval & Group A [pain pump] & Group B [saline pump] & Group C [no pump] \\
8 hour post-op & 2.5 & 1.8 & 0.3282 \\
At 24 hours & 1.75 & 3.23 & 3.06 \\
At 48 hours & 1.81 & 3.17 & 0.16 \\
At 72 hours & 3.3 & 4.05 & 0.14 \\
\hline
\end{tabular}

surgery, a significant difference was noted in the amount of pain medication required after discharge from the recovery room in Group C (no pain pump), compared to the other two groups $(\mathrm{p}=0.03)$. There was no difference in Group A (pain pump) or Group B (saline pump) during this time interval. There was no significant difference noted in pain scores among the three groups for all other time intervals during the study. The total narcotics utilized by each group are shown (Table 3). Group B (saline pump) had higher narcotic use than other two groups on post op day 2 but the value did not reach statistical significance. At the most recent follow-up, all patients had a subjective pain score of 2 or less.

Patient knee range of motion was analyzed at postoperative weeks 1 and 6 (Table 4). When knee range of motion was compared pre-operatively and at 1 and 6 weeks post-operatively, no significant difference was found for the entire group. When pre-operative knee range of motion was compared at weeks 1 and 6 among the 3 groups, no statistical difference was noted.

Demographic factors including body mass index, smoking, sex, race, age and previous surgeries were analyzed in relation to VAS pain scores and amount of narcotics used after surgery. No statistical relationship was noted.

Due to recent reports in the orthopaedic literature describing chondrolysis associated with the use of a continuous flow pain pump, at a minimum of 12 months post-operatively, an attempt was made to contact all study patients for follow-up. Twenty-nine patients were available for clinical and radiographic examination or a phone interview. Among these patients, the average follow-up was 18 months, with four patients undergoing a total or unicondylar knee arthroplasty. Upon review of the 4 subjects' intra-operative arthroscopy photographs, all patients had evidence of partial or full thickness cartilage damage in at least 1 compartment at the time of surgery. Two additional patients indicated that their arthritis has progressed since surgery but that their symptoms were not severe enough to warrant further intervention. The patients' pre-operative and most recent postoperative radiographic scores were analyzed using a paired t-test. No significant difference was noted for scores for any group [Group A, p = 0.34; Group B, p = 0.1 ; Group $\mathrm{C}, \mathrm{p}=1.0]$. ANOVA was used to analyze whether there was any difference between the groups and no significant difference was found $[\mathrm{p}=0.11]$.

\section{Discussion}

The concept of multimodal analgesia was introduced to improve analgesia and reduce the incidence of opioidrelated adverse events. It is achieved by combining different analgesics, resulting in synergistic analgesia with lowered adverse effects [7]. The literature varies on the most effective combination, dosage or method of administration.

Several studies have examined the beneficial effects of combining multiple medications in a single intraarticular dose after arthroscopic knee surgery. Jaureguito et al. [8] noted a benefit with the use of intraarticular morphine when used post-operatively in conjunction with bupivacaine. Gupta et al. found the combination of ketorolac plus morphine to be more effective in relieving postoperative pain after knee arthroscopy than ketorolac or morphine alone [9]. Calmet et al. reported $60 \mathrm{mg}$ of ketorolac administered after knee arthroscopy to be superior to $10 \mathrm{~mL}$ of $0.25 \%$ bupivacaine or $1 \mathrm{mg}$ of morphine [10]. Most previous studies evaluating post-operative analgesia have utilized bupivacaine alone or in combina- 
Table 3. Mean number of narcotic pills and morphine sulfate used for the 3 groups at different time intervals.

\begin{tabular}{ccccc}
\hline Time interval & Group A [pain pump] & Group B [saline pump] & Group C [no pump] & p value \\
\hline Narcotic use in recovery room & 2.2 & 1.8 & 1.3 & 0.61 \\
Narcotic medication post discharge & 1.18 & 2.0 & 2.4 & $0.03^{*}$ \\
Total narcotic on post-op day \#1 (pills) & 3.41 & 3.8 & 0.8 & 0.87 \\
Total narcotic on post-op day \#2 (pills) & 1.1 & 1.5 & 1.7 & 0.08 \\
Total narcotic on post-op day \#3 (pills) & 2.1 & 2.7 & 0.16 \\
\hline
\end{tabular}

*Post hoc analysis showed Group B significantly different from Groups A and C.

Table 4. Knee range of motion for the 3 groups pre-operatively and post-operatively at weeks 1 and 6.

\begin{tabular}{ccccc}
\hline & Group A & Group B & Group C & p value \\
\hline Pre-op & 120 & 120 & 122 & 0.1 \\
Week 1 & 106 & 117 & 105 & 0.17 \\
Week 6 & 116 & 129 & 126 & 0.14 \\
\hline
\end{tabular}

tion with other medicines. In the present study, ropivacaine was chosen due to its clinical efficacy and diminished cardiac and chondrocyte toxicity $[11,12]$.

Continuous infiltration of local anesthetics for postoperative pain control has been used in the past with mixed outcomes [5,13-17]. There have been limited studies evaluating the effect of a continuous intraarticular pain pump after arthroscopic knee surgery [13,14,18].

In the current study, intraarticular, subcutaneous and intramuscular medicine infiltration was used. Townshend et al. [19] noted equivalent efficacy between portal and intraarticular anesthetic administration following knee arthroscopy. The authors suggested that portal infiltration might be an important component of post-operative pain management.

The current study noted no difference in pain scores or narcotic use the day after surgery for patients with or without a pain pump. Our data is in contrast to the findings of Hoenecke et al. [14] and Chew et al. [13] who reported a beneficial effect of an infusion pump after anterior cruciate ligament reconstruction. However, the authors used only a single bolus medication, did not include a group without a pain catheter and did not address the placebo effect of a catheter. In the current study, significantly more narcotic medication was utilized after discharge from the recovery room the day of surgery in patients in Group C (no pain pump), compared to the other two groups $(p=0.03)$. This finding is suggestive of placebo effect in Group B that is well documented in published knee injection studies [20,21].

Various in vitro studies have demonstrated bupivacaine and lidocaine to be toxic to chondrocytes [22-24]. Grishko et al. noted ropivacaine to be significantly less toxic at lower doses [25]. Currently, questions remain about the relationship between chondrolysis and the use of continuous intraarticular local anesthetics. Although more frequently reported in the shoulder, a recent series of three cases of chondrolysis was published with the use of an intraarticular pain pump after knee arthroscopy [26-30]. Many surgeons have abandoned the use of continuously infused local anesthetics, but continue to use the drugs as a one-time injection.

The purpose of the current study was to evaluate the efficacy of an intraarticular and subcutaneous injection alone in comparison to an identical injection plus an intra-articular pump. A secondary objective was to evaluate the safety of continuous intra-articular ropivacaine administered for 24 hours. In light of a recent recommendation that the term chondrolysis be applied only to patients who have had an operative intervention within the previous 12 months, the authors elected to evaluate the study groups at a minimum of 12 months post-operatively [31]. The hallmarks of chondrolysis are radiographic joint space narrowing within the first 12 months after surgery and progressive unrelenting pain. In the current study at an average of 18 months post-operatively, no patient demonstrated clinical or radiographic evidence of chondrolysis after the use of a ropivacaine pain pump. 
Our study has several weaknesses. The first is the use of two different pain pumps. Due to a United States Justice Department investigation of the initial pain pump manufacturer, this pump became unavailable. The study was temporarily halted until our Institutional Review Board approved an identical pump from a different manufacturer. The authors believe that the identical pressure, flow rate and volume of each device enabled the study to continue without compromise. The second weakness is the lack of follow-up in the evaluation of chondrolysis. Despite arduous attempts by phone and internet, the authors were only able to contact $60 \%$ of the study patients at the 12 months follow-up. Although, a larger percentage of the subjects were evaluated prior to 12 months, the authors were unable to contact the remaining subjects at the 12 months time interval. No chondrolysis was observed clinically or radiographically in any study patient at any time; however, there exists the possibility that some of these patients developed chondrolysis in the interval between their last follow-up and 12 months postoperatively.

In conclusion, a single subcutaneous and intraarticular injection of ketorolac, morphine and ropivacaine was as effective as this solution plus a 24-hour intraarticular ropivacaine pain pump for post-operative pain relief after outpatient arthroscopic knee surgery. No clinical or radiographic signs of chondrolysis were seen in the knee with the use of intraarticular ropivacaine at an average of 18 months post-operatively.

\section{REFERENCES}

[1] N. N. Basu, B. Kald and D. Heath, "Morphine Delays Discharge Following Ambulatory Surgery: A Prospective Institutional Study," Journal of Perioperative Practice, Vol. 19, No. 8, 2009, pp. 254-256.

[2] K. A. Faccenda and B. T. Finucane, "Complications of Regional Anaesthesia Incidence and Prevention," Drug Safety, Vol. 24, No. 6, 2001, pp. 413-442. doi:10.2165/00002018-200124060-00002

[3] P. Essving, K. Axelsson, J. Kjellberg, O. Wallgren, A. Gupta and A. Lundin, "Reduced Morphine Consumption and Pain Intensity with Local Infiltration Analgesia (LIA) Following Total Knee Arthroplasty," Acta Orthopaedica, Vol. 81, No. 3, 2010, pp. 354-360. doi:10.3109/17453674.2010.487241

[4] P. Essving, K. Axelsson, J. Kjellberg, O. Wallgren, A. Gupta and A. Lundin, "Reduced Hospital Stay, Morphine Consumption, and Pain Intensity with Local Infiltration Analgesia after Unicompartmental Knee Arthroplasty," Acta Orthopaedica, Vol. 80, No. 2, 2009, pp. 213-219. doi:10.3109/17453670902930008

[5] P. Gómez-Cardero and E. C. Rodríguez-Merchán, "Postoperative Analgesia in TKA: Ropivacaine Continuous Intraarticular Infusion," Clinical Orthopaedics and Related Research, Vol. 468, No. 5, 2010, pp. 1242-1247. doi:10.1007/s11999-009-1202-2

[6] F. A. Barber and M. A. Herbert, "The Effectiveness of an Anesthetic Continuous-Infusion Device on Postoperative Pain Control," Arthroscopy, Vol. 18, No. 1, 2002, pp. 7681. doi:10.1053/jars.2002.25976

[7] H. Kehlet and J. B. Dahl, "The Value of 'Multimodal' or 'Balanced Analgesia' in Postoperative Pain Treatment," Anesthesia \& Analgesia, Vol. 77, No. 5, 1993, pp. 10481056. doi:10.1213/00000539-199311000-00030

[8] J. W. Jaureguito, J. F. Wilcox, S. J. Cohn, R. A. Thisted and B. Reider, "A Comparison of Intraarticular Morphine and Bupivacaine for Pain Control after Outpatient Knee Arthroscopy. A Prospective, Randomized, Double-Blinded Study," The American Journal of Sports Medicine, Vol. 23, No. 3, 1995, pp. 350-353. doi: $10.1177 / 036354659502300318$

[9] A. Gupta, K. Axelsson, R. Allvin, J. Liszka-Hackzell, N. Rawal, B. Althoff and B. G. Augustini, "Postoperative Pain Following Knee Arthroscopy: The Effects of IntraArticular Ketorolac and/or Morphine," Regional Anesthesia and Pain Medicine, Vol. 24, No. 3, 1999, pp. 225-230. doi:10.1016/S1098-7339(99)90132-3

[10] J. Calmet, C. Esteve, S. Boada and J. Giné, "Analgesic Effect of Intra-Articular Ketorolac in Knee Arthroscopy: Comparison of Morphine and Bupivacaine," Knee Surgery, Sports Traumatology, Arthroscopy, Vol. 12, No. 6, 2004, pp. 552-555. doi:10.1007/s00167-003-0483-3

[11] H. S. Feldman, "Toxicity of Local Anaesthetic Agents," In: S. Rice and K. Fish, Eds., Anesthetic Toxicity, Raven Press, New York, 1994, pp. 107-133.

[12] D. D. Denson, J. A. Myers, C. T. Hartrick, C. P. Pither, D. E. Coyle and P. P. Raj, "The Relationship between Free Bupivacaine Concentration and Central Nervous System Toxicity," Anesthesiology, Vol. 61, No. 3, 1984, p. A211. doi:10.1097/00000542-198409001-00211

[13] H. F. Chew, N. A. Evans and W. D. Stanish, "PatientControlled Bupivacaine Infusion into the Infrapatellar Fat Pad after Anterior Cruciate Ligament Reconstruction," Arthroscopy, Vol. 19, No. 5, 2003, pp. 500-505. doi: $10.1053 /$ jars. 2003.50110

[14] H. R. Hoenecke, P. A. Pulido, B. A. Morris and J. Fronek, "The Efficacy of Continuous Bupivacaine Infiltration Following Anterior Cruciate Ligament Reconstruction," Arthroscopy, Vol. 18, No. 8, 2002, pp. 854-858. doi:10.1053/jars.2002.36139

[15] M. Bianconi, L. Ferraro, G. C. Traina, G. Zanoli, T. Antonelli, A. Guberti, R. Ricci and L. Massari, "Pharmacokinetics and Efficacy of Ropivacaine Continuous Wound Instillation after Joint Replacement Surgery," British Journal of Anaesthesia, Vol. 91, No. 6, 2003, pp. 830-835. doi:10.1093/bja/aeg277

[16] F. T. DeWeese, Z. Akbari and E. Carline, "Pain Control after Knee Arthroplasty: Intraarticular versus Epidural Anesthesia," Clinical Orthopaedics and Related Research, Vol. 392, 2001, pp. 226-231. doi:10.1097/00003086-200111000-00028

[17] J. Nechleba, V. Rogers, G. Cortina, and T. Cooney, “Continuous Intra-Articular Infusion of Bupivacaine for Postoperative Pain Following Total Knee Arthroplasty," The 
Journal of Knee Surgery, Vol. 18, No. 3, 2005, pp. 197202.

[18] J. W. Alford and P. D. Fadale, "Evaluation of Postoperative Bupivacaine Infusion for Pain Management after Anterior Cruciate Ligament Reconstruction," Arthroscopy, Vol. 19, No. 8, 2003, pp. 855-861. doi:10.1016/S0749-8063(03)00734-5

[19] D. Townshend, K. Emmerson, S. Jones, P. Partington and S. Muller, "Intra-Articular Injection versus Portal Infiltration of $0.5 \%$ Bupivacaine Following Arthroscopy of the Knee: A Prospective, Randomised Double-Blinded Trial," The Journal of Bone and Joint Surgery, Vol. 91, No. 5, 2009, pp. 601-603. doi:10.1302/0301-620X.91B5.21932

[20] R. D. Altman and R. Moskowitz, "Intraarticular Sodium Hyaluronate (Hyalgan) in the Treatment of Patients with Osteoarthritis of the Knee: A Randomized Clinical Trial. Hyalgan Study Group," The Journal of Rheumatology, Vol. 25, No. 11, 1998, pp. 2203-2212.

[21] E. C. Huskisson and S. Donnelly, "Hyaluronic Acid in the Treatment of Osteoarthritis of the Knee," Rheumatology (Oxford), Vol. 38, No. 7, 1999, pp. 602-607. doi:10.1093/rheumatology/38.7.602

[22] C. R. Chu, C. H. Coyle, C. T. Chu, M. Szczodry, V. Seshadri, J. C. Karpie, K. M. Cieslak and E. K. Pringle, "In Vivo Effects of Single Intra-Articular Injection of $0.5 \%$ Bupivacaine on Articular Cartilage," The Journal of Bone and Joint Surgery. American Volume, Vol. 92, No. 3, 2010, pp. 599-608. doi:10.2106/JBJS.I.00425

[23] J. L. Dragoo, T. Korotkova, R. Kanwar and B. Wood, "The Effect of Local Anesthetics Administered via Pain Pump on Chondrocyte Viability," The American Journal of Sports Medicine, Vol. 36, No. 8, 2008, pp. 1484-1488. doi: $10.1177 / 0363546508318190$

[24] C. R. Chu, N. J. Izzo, C. H. Coyle, N. E. Papas and A. Logar, "The in Vitro Effects of Bupivacaine on Articular Chondrocytes," The Journal of Bone and Joint Surgery. British Volume, Vol. 90, No. 6, 2008, pp. 814-820.

\section{doi:10.1302/0301-620X.90B6.20079}

[25] V. Grishko, M. Xu, G. Wilson and A. W. Pearsall, “Apoptosis and Mitochondrial Dysfunction in Human Chondrocytes Following Exposure to Lidocaine, Bupivacaine, and Ropivacaine," The Journal of Bone and Joint Surgery. American Volume, Vol. 92, No. 3, 2010, pp. 609-618. doi:10.2106/JBJS.H.01847

[26] J. M. Malinovsky and H. Ludot, "Severe Chondrolysis after Shoulder Arthroscopy: A Case Series," Annales Françaises d'Anesthésie et de Réanimation, Vol. 28, No. 11, 2009, pp. 999-1000.

[27] P. P. Mariani, R. Garofalo and F. Margheritini, "Chondrolysis after Partial Lateral Meniscectomy in Athletes," Knee Surgery, Sports Traumatology, Arthroscopy, Vol. 16 , No. 6, 2008, pp. 574-580. doi:10.1007/s00167-008-0508-Z

[28] J. H. Rapley, R. C. Beavis and F. A. Barber, "Glenohumeral Chondrolysis after Shoulder Arthroscopy Associated with Continuous Bupivacaine Infusion," Arthroscopy, Vol. 25, No. 12, 2009, pp. 1367-1373. doi:10.1016/j.arthro.2009.08.024

[29] P. T. Scheffel, J. Clinton, J. R. Lynch, W. J. Warme, A. L. Bertelsen and F. A. Matsen, "Glenohumeral Chondrolysis: A Systematic Review of 100 Cases from the English Language Literature," Journal of Shoulder and Elbow Surgery, Vol. 19, No. 6, 2010, pp. 944-949. doi:10.1016/i.jse.2010.01.023

[30] E. W. Fester and F. R. Noyes, "Postoperative Chondrolysis of the Knee: 3 Case Reports and a Review of the Literature," The American Journal of Sports Medicine, Vol. 37, No. 9, 2009, pp. 1848-1854. doi: $10.1177 / 0363546509334999$

[31] M. T. Provencher, M. Navaie, D. J. Solomon, J. C. Smith, A. A. Romeo and B. J. Cole, "Joint Chondrolysis," The Journal of Bone and Joint Surgery. American Volume, Vol. 93, No. 21, 2011, pp. 2033-2044. doi:10.2106/JBJS.J.01931 